\title{
Antibiotics are not useful to reduce pain associated with irreversible pulpitis
}

\author{
Abstracted from \\ Agnihotry A, Fedorowicz Z, van Zuuren EJ, Farman AG, Al-Langawi JH. \\ Antibiotic use for irreversible pulpitis. Cochrane Database Syst Rev 2016; 2: Art. No. CD004969. \\ DOI:10.1002/14651858.CD004969.pub4. \\ Address for correspondence: Anirudha Agnihotry, Section of Restorative Dentistry, UCLA School of Dentistry, \\ 10833 Le Conte Avenue, Los Angeles, CA 90095-1668, USA. E-mail: anirudha.agnihotry@gmail.com
}

\section{Question: Do antibiotics used preoperatively reduce tooth pain associated with irreversible pulpitis?}

Data sources Cochrane Oral Health Group's Trials Register, the Cochrane Central Register of Controlled Trials, MEDLINE, EMBASE, Clinical Trials.gov and the WHO International Clinical Trials Registry Platform. There were no language restrictions.

Study selection Randomised controlled trials which compared the relief of pain with systemic antibiotics and analgesics against placebo and analgesics in the preoperative phase of irreversible pulpitis. The primary interest was pain control with an antibiotic or without one in the presence of analgesics. The secondary outcomes were type, dose and frequency of medication for pain relief and any adverse effects related to hypersensitivity or other reactions to either the antibiotic or analgesics.

Data extraction and synthesis Two authors independently assessed the results of the searches. Data extraction and risk bias assessment were also carried out independently. A third reviewer settled any disagreement on inclusion. Since only one study was included a metaanalysis could not be performed.

Results Only one double blind randomised clinical trial involving 40 participants with a diagnosis of irreversible pulpitis in one of their teeth was included in this review. This was a low risk, well-constructed double blind study. Half of the participants were treated with penicillin $500 \mathrm{mg}$, the other with a placebo every six hours over a seven- day period. In addition, all the participants were instructed to initially take one tablet of ibuprofen every 4-6 hours as needed and to take acetaminophen with codeine (two tablets every 4-6 hours) only if the ibuprofen did not relieve the pain.

There was no significant difference in the mean total number of ibuprofen tablets over the study period; 9.2(standard deviation (SD) 6.02) in the penicillin group versus, 9.6 (SD 6.34) in the placebo group; mean difference $-0.40(95 \% \mathrm{Cl}-4.23$ to 3.43$)$; $P$ value $=0.84$. The mean total number of Tylenol tablets, 6.9 (SD 6.87), used in the

This paper is based on a Cochrane Review published in the Cochrane Library 2016, issue 2 (see www.thecochranelibrary.com for information). Cochrane Reviews are regularly updated as new evidence emerges and in response to feedback, and the Cochrane Library should be consulted for the most recent version of the review. penicillin group versus 4.45 (SD4.42) in the placebo group; mean difference $2.45(95 \% \mathrm{Cl}-1.23$ to 6.13 ; P value $=0.19)$. The study did not address adverse events.

Conclusions There is insufficient evidence to accept or refute the benefit of using penicillin to control pain intensity associated with irreversible pulpitis. This study primarily found that antibiotics given preoperatively (before definitive root canal therapy) do not appear to significantly reduce toothache caused by irreversible pulpitis. Between the two groups, those taking the antibiotics and those taking the placebo, there was no statistical difference in the amount of oral analgesics taken to relieve the pain.

\section{Commentary}

The American Association of Endodontists (AAE) defines symptomatic irreversible pulpitis as a clinical diagnosis based on subjective and objective findings indicating that the vital inflamed pulp is incapable of healing. ${ }^{1}$ Additional descriptors for this condition include lingering thermal pain, spontaneous pain and referred pain. Some describe this type of pain's intensity as 'worse than childbirth'. Given this information one can understand why there aren't many studies like this one conducted to be included in this review. Other studies that were excluded were ones where antibiotics were used either during or after endodontic therapy was initiated. Ethically can a researcher withhold treatment, for seven days, that we know will ultimately eliminate extreme pain in order to test a hypothesis?

This second Cochrane systematic review updates a previous review published in 2013 that concluded that there was insufficient evidence to determine whether penicillin is effective in relieving the pain from irreversible pulpitis. The driving force for this renewed interest may be a dire need to establish best practices when it comes to antibiotic usage. As mentioned in this Cochrane review, the US Center for Disease Control and Prevention estimates that 50 million courses of antibiotics are given without necessity. ${ }^{2}$ It is well known that this overuse of antibiotics can lead to the increase in infections due to multi-drug resistant bacteria.

By definition the pain from irreversible pulpitis is caused by an inflamed vital pulp, not an infected non-vital pulp, therefore not only is an antibiotic not going to cause a decrease in pain, it isn't going to treat an infection that is not present. In addition to this in vital pulps with a normal blood supply only a small amount of antibiotic ever reaches the pulp. ${ }^{3}$ 
In the only study included in the review, participants were asked to assess their pain each morning on a scale from 0-3 where 0 indicates no pain and 3 is severe pain. They were also asked to rate their pain on tooth percussion by tapping the affected tooth with their finger. The study participants used sum of pain intensity difference (SPID) weighted by the length of the interval since the previous observation and sum of pain percussion intensity difference (SPPID) also weighted by the length of the interval since the previous observation to record the pain changes from day to day. The distributions of the pain ratings in both groups were close. The SPID for the penicillin group was $6.0 \pm 10.5$ and for the placebo $6.0 \pm 9.5 \mathrm{P}$ value $=0.776$ and the SPPID for the penicillin group was $3.5 \pm 7.5$ and placebo $2.0 \pm 7.0$ $\mathrm{P}$ value $=0.290$. These were considered statistically significant at $P$ value $>0.05$.

This systematic review may have low quality of evidence in that it contains only one study with (only 40 participants) but, given the science of irreversible pulpitis and therefore the lack of necessity to prescribe an antibiotic (when an infection is not present) in conjunction with this study that shows that there is insufficient evidence to point to its effectiveness in relieving pain, the evidence to recommend a discontinuance of antibiotic use for this purpose is strong. Three years and many millions of dosages of antibiotics that cause resistant strains of bacteria later isn't it time to put an end to this misguided practice of antibiotic misuse and recommend that the prescribing of antibiotics for pain from irreversible pulpitis abate?

Antibiotics may decrease pain due to facial swelling from acute apical abscesses that are indeed due to bacterial infections but they are definitely not indicated for tooth pain relief due to an irreversible pulpitis.

Eileen Hoskin ${ }^{\mathrm{a}}$ and Analia Veitz-Keenan ${ }^{\mathrm{b}}$

${ }^{a}$ Rutgers School of Dental Medicine, New Jersey, USA

${ }^{b}$ New York University, College of Dentistry, New York, USA

1. www.aae.org/uploadedfiles/publications_and_research/ newsletters/endodontics_colleagues_for_excellence_newsletter/ aaeconsensusconferencerecommendeddiagnosticterminology.pdf [Accessed 15 August 2016]

2. Colgan R, Powers JH. Appropriate antimicrobial prescribing: approaches that limit antibiotic resistance. Am Fam Physician 2001; 64: 999-1004.

3. AkimitoY, Kaneko K, Fujii A, Tamura T. Ampicillin concentrations in human serum, gingiva, mandibular bone, dental follicle, and dental pulp following a single oral use of talampicillin. J Oral Maxillofac Surg 1985; 43: 270-276.

Evidence-Based Dentistry (2016) 17, 81-82. doi:10.1038/sj.ebd.6401187 